\title{
SOCIOECONOMIC STATUS AND ADVERSE BIRTH OUTCOMES: A POPULATION-BASED CANADIAN SAMPLE
}

\author{
EMILY E. CAMPBELL*, JASON GILLILAND†, \\ PAULA D. N. DWORATZEK*, BARBRA DE VRIJER $\$$, DEBBIE PENAVA \\ AND JAMIE A. SEABROOK*1 \\ *School of Food and Nutritional Sciences, Brescia University College, London, Ontario, \\ Canada, †Department of Geography, Western University, London, Ontario, \\ Canada and $\$$ Department of Obstetrics and Gynaecology, Western University, London, \\ Ontario, Canada
}

\begin{abstract}
Summary. This study assessed the strength of the association between socioeconomic status (SES) and low birth weight (LBW) and preterm birth (PTB) in Southwestern Ontario. Utilizing perinatal and neonatal databases at the London Health Science Centre, maternal postal codes were entered into a Geographic Information System to determine home neighbourhoods. Neighbourhoods were defined by dissemination areas (DAs). Median household income for each DA was extracted from the latest Canadian Census and linked to each mother. All singleton infants born between February 2009 and February 2014 were included. Of 26,654 live singleton births, 6.4\% were LBW and 9.7\% were PTB. Top risk factors for LBW were: maternal amphetamine use, chronic hypertension and maternal marijuana use (OR respectively: $17.51,3.18,2.72$ ); previously diagnosed diabetes, maternal narcotic use and insulin-controlled gestational diabetes predicted PTB (OR respectively: $17.95,2.69,2.42)$. Overall, SES had little impact on adverse birth outcomes, although low maternal education increased the likelihood of a LBW neonate (OR: 1.01).
\end{abstract}

\section{Introduction}

Socioeconomic status (SES) is a measure of an individual's position within a hierarchical social structure. The three most common indicators of SES are household income, education and occupation. Other measures include neighbourhood income, family structure, race/ethnicity and the accumulation of assets or wealth (Daly et al., 2002; Seabrook \& Avison, 2015). Socioeconomic status is a consistent and reliable predictor of health disparities, as socioeconomically disadvantaged groups tend to have poorer

${ }^{1}$ Corresponding author: Email: jseabro2@uwo.ca 
physical and mental health than their high-SES counterparts (O'Rand \& Hamil-Luker, 2005; Haas, 2008; Seabrook \& Avison, 2010). Low-SES individuals also experience greater exposure to stress, and are more likely to engage in risk-taking behaviours such as smoking and alcohol/drug abuse (Seabrook \& Avison, 2012). Moreover, low-SES groups are more likely to be overweight, and are less likely to get regular exercise, both of which contribute to a decreased health status (Lynch et al., 1997).

One strategy to decrease the socioeconomic gradient in health disparities is to minimize adverse events that occur in utero and which impact birth outcomes. Birth weight is a marker of fetal growth, and is linked to maternal health before and during pregnancy. It is also an indicator of a newborn's health status and is a determinant of infant growth, development and survival (Williams et al., 2000). While low birth weight (LBW) and preterm birth (PTB) are related, they have individual aetiologies and manifestations. These pregnancy outcomes represent a serious public health problem and lead to high health care service costs for children and families (Williams et al., 2000; Zeitlin et al., 2001; Siqueira et al., 2007). Low birth weight is associated with elevated rates of respiratory problems and asthma, and poor cognitive development during infancy and childhood (Boardman et al., 2001). Later-life health consequences include type 2 diabetes, hypertension and cardiovascular disease (World Health Organization, 2014; Barker, 2004). Conversely, PTB increases an infant's risk for childhood neurologic disability and long-term medical consequences including respiratory diseases, gastrointestinal disorders, cardiovascular disease and decreased immunity (FoulderHughes \& Cooke, 2003; Hofman et al., 2004; Tucker \& McGuire, 2004; Behrman \& Butler, 2007). Both PTB and LWB are associated with a higher risk of infant morbidity and mortality (Dunn, 1984; Kramer et al., 2001). Additionally, education, income, neighbourhood SES and occupation are independently associated with birth outcomes, including PTB and LBW (Campbell \& Seabrook, 2016).

There have been few population-based studies investigating the relationship between SES and birth outcomes in Canada, as birth registrations differ between provinces and territories with regards to the type of data collected, and access to perinatal databases can be challenging for population health researchers (Joseph et al., 2007; Luo et al., 2004, 2006). Additionally, the majority of studies investigating the association between SES and birth outcomes have been conducted in the United States, and it is unclear to what extent these results can be generalized to Canada due to differences in the socioeconomic gradient and health care systems between the two countries. Compared with the United States, Canada has smaller income disparities, and all Canadians have access to basic health care. It has been suggested that, relative to the United States, the smaller income inequality in Canada, coupled with its universal health care system, may attenuate the relationship between SES and disease (Ross et al., 2000; Willson, 2009).

In the few studies that have been conducted in Canada, a moderate socioeconomic association with birth outcomes has been found. Luo et al. (2004) found that women living in the lowest neighbourhood income quintile in rural (OR: 1.03; 95\% CI: 1.01, 1.04) and urban (OR: 1.04; 95\% CI: 1.03, 1.05) British Columbia had a moderately greater risk of giving birth to a preterm infant compared with women in the highest income quintile. However, important risk factors such as smoking and alcohol/drug use were not captured in the study, and it plausible that these factors could confound the association between neighbourhood income and birth outcomes. Similar results were 
found in a study in Quebec, where women in the lowest income quintile were more likely to experience a PTB (OR: 1.14; 95\% CI: 1.10, 1.17) (Luo et al., 2006). Heaman et al. (2013) found that low levels of education (less than grade 12), having a previous medical condition, developing a new medical condition or health problem during pregnancy, being a primigravida or being a multigravida with a previous PTB or a previous miscarriage or abortion were associated with PTB. The objective of the current study was to determine the strength of the relationship between SES and adverse birth outcomes, particularly LBW and PTB, after controlling for epidemiological and medical risk factors known to be associated with adverse birth outcomes.

\section{Methods}

Study sample

This retrospective cohort study utilized a large, population-based sample from Southwestern Ontario to investigate the relationship between socioeconomic status and LBW and PTB. Data were obtained from the perinatal and neonatal databases at the London Health Science Centre (LHSC), a tertiary care facility whose catchment area includes approximately 1.5 million patients per year. Data for all births at the LHSC were prospectively entered from medical charts, and birth and neonatal records were recorded by a dedicated research assistant (Lackman et al., 2001). Using maternal postal codes as a geographic identifier, the database was linked with neighbourhood-level SES variables. Specifically, maternal postal codes were entered into a Geographic Information System (ArcGIS 10.1, Environmental Systems Research Institute, Redlands, CA) and mapped to determine location of home neighbourhoods. Boundaries of home neighbourhoods were defined by boundaries of census dissemination areas (DAs), a commonly used proxy for neighbourhood and the smallest geographical unit for which Statistics Canada releases income data (Healy \& Gilliland, 2012).

Data on neighbourhood-level socioeconomic variables for each DA in Southwestern Ontario, the catchment area of the LHSC, were extracted from the latest Canadian Census (Statistics Canada, 2011) and linked to each mother. Neighbourhood-level (DA) socioeconomic status variables included: (1) maternal education: the percentage of the female population between the ages of 25 and 64 years in the DA (in 2011) with highest level of education as either a high school diploma or no high school diploma (compared with the total female population aged 25-64 years in the DA); (2) low-income cut-off (LICO): the percentage of the DA population (in 2010) who live under the low-income cut-off (Statistics Canada defines LICOs as income thresholds below which a family is likely to devote a larger share of its income to the necessities of shelter, food and clothing than the average family); (3) recent immigration prevalence: the percentage of the DA population that immigrated to Canada between 2006 and 2011; (4) visible minority prevalence: the percentage of the DA population that self-identify as a visible minority; (5) Aboriginal prevalence: the percentage of the DA population that self-identify as Aboriginal identity; and (6) distance to grocery store: the distance between postal code centroid and the nearest grocery store in kilometres.

All singleton infants without congenital anomalies who were born between February 2009 and February 2014 at the LHSC were included in the study. Low birth weight 
infants were classified as a birth weight less than $2500 \mathrm{~g}$. Preterm birth was defined as a live birth of less than 37 weeks' gestational age. The final sample consisted of 26,654 live births.

This study received approval from the Research Ethics Board at Western University (File No.: 105968) and the Lawson Health Research Institute at the LHSC in London, Canada.

\section{Statistical analysis}

Data analysis was conducted using SPSS version 23 (IBM SPSS Statistics for Windows). Descriptive statistics are presented as mean \pm standard deviation (SD) or median and interquartile range (IQR) at the $25^{\text {th }}$ and $75^{\text {th }}$ percentile to summarize demographic, socioeconomic and clinical characteristics. Chi-square tests were used to determine the association between categorical prenatal risk factors and LBW and PTB, and univariate logistic regressions were used to assess the relationship between continuous independent variables and the two birth outcomes. To test for multicollinearity, a correlation matrix was used to assess the strength of the association between independent variables, where any strong correlations $(r \geq \pm 0.80)$ were considered a sign of possible collinearity. However, no correlation coefficients were close to approaching a strong association. Intraclass correlations (ICCs) were also used to assess the extent to which observations in the same DA were more related than observations from different DAs, and the ICCs were close to 0 , indicating that the observations were independent of each other. Therefore, multilevel analyses were rejected in favour of traditional logistic regression. All univariate associations that had a significant relationship with LBW and/or PTB $(p<0.05)$ were subsequently included in multivariable regression models. Separate logistic regressions were computed with LBW and PTB as dependent variables. The strength of the association between SES and LBW/PTB was determined through odds ratios (ORs) from the final multivariable logistic regression. A two-sided $p$-value $<0.05$ was considered statistically significant.

Utilizing percentile growth curves developed by Kramer et al. (2001), a secondary analysis was computed by classifying infants based on sex-specific, population-based references developed in Canada. This analysis was conducted to assess the influence of birth percentile $\left(<5^{\text {th }}\right.$ percentile and $<10^{\text {th }}$ percentile $)$ on LBW and PTB.

\section{Results}

Out of a total of 26,654 live singleton births between February 2009 and February 2014, $6.4 \%$ were LBW and $9.7 \%$ were PTB. The mean age of mothers was $33.8 \pm 5.6$ years. The mean gestational age at birth was $38.8 \pm 5.4$ weeks, and the mean birth weight was $3349.2 \pm 665.3 \mathrm{~g}$, with $79.9 \%$ of the sample falling between 2500 and $3999 \mathrm{~g}$. The mean maternal pre-pregnancy body mass index (BMI) was $25.5 \pm 6.2 \mathrm{~kg} / \mathrm{m}^{2}$. Further demographic, socioeconomic and clinical characteristics of the sample are shown in Tables 1 and 2.

Unadjusted analyses (data not shown) showed that women who gave birth to a LBW infant were more likely to have had a previous PTB; to have fewer previous live births; a diagnosis of chronic hypertension; lower pre-pregnancy BMI; a history of smoking tobacco and/or marijuana use; mental illness and/or previous anxiety. Additionally, these LBW infants had a lower gestational age and were more likely to be female. 
Table 1. Selected demographic variables among women who had singleton live births at the LHSC between February 2009 and February 2014, $N=26,654$

\begin{tabular}{lc}
\hline & Mean \pm SD/Median (IQR) \\
\hline Maternal characteristics (mean $\pm \mathrm{SD})$ & \\
Maternal age (years) & $33.8 \pm 5.6$ \\
Gestational age at birth (weeks) & $38.8 \pm 5.4$ \\
Maternal BMI $\left(\mathrm{kg} / \mathrm{m}^{2}\right)$ & $25.5 \pm 6.2$ \\
Birth weight $(\mathrm{g})$ & $3349.2 \pm 665.3$ \\
Sex of infant & \\
Female $(n=12,703)$ & 49.1 \\
Male $(n=13,164)$ & 50.9 \\
Number of previous abortions & $0.5 \pm 0.9$ \\
Number of previous live births & $0.9 \pm 1.4$ \\
Neighbourhood characteristics (median (IQR)) & \\
Distance to grocery store $(\mathrm{km})$ & $0.9(0.3,10.0)$ \\
Visible minority prevalence $(\%)$ & $7.6(0.0,19.5)$ \\
Immigration prevalence $(\%)$ & $0.0(0.0,1.70)$ \\
Aboriginal prevalence $(\%)$ & $0.0(0.0,1.2)$ \\
<High school diploma $(\%)$ & $19.8(12.4,26.3)$ \\
After-tax LICO 2010 & $10.6(4.5,22.3)$ \\
\hline
\end{tabular}

${ }^{\mathrm{a}} \mathrm{LICO}$ : low-income cut-off.

Socioeconomic status variables that were statistically significant $(p<0.05)$ included neighbourhood income, recent immigration prevalence (2006-2011), distance to grocery store, maternal education, lower 2010 after-tax income and lone-parent household.

Women who gave birth to a PTB infant were more likely to have had a previous PTB; a diagnosis of chronic hypertension, cardiac condition, anxiety during this pregnancy and/or diabetes; and not to have had reproductive technology. Again, these PTB infants were lower birth weight and more likely to be female. Socioeconomic status variables that were statistically significant included neighbourhood income, distance to grocery store, immigration prevalence, visible minority prevalence, maternal education and lower 2010 after-tax income. Interestingly, maternal age was not a significant predictor of either LBW or PTB.

Results from the logistic regression models can be found in Table 3. For LBW, significant variables included higher number of previous live births, previous PTB, lower gestational age at birth, chronic hypertension, previously diagnosed maternal diabetes, female infant, maternal marijuana and/or amphetamine use, smoking tobacco and lower maternal BMI. Maternal smoking and drug use were measured as yes/no outcomes, and did not include regular or occasional use. Maternal amphetamine use had the largest relative influence on LBW (OR: 17.51; 95\% CI: 1.45, 211.04). Maternal education had a weak, but statistically significant association with LBW in the final logistic regression. Mothers with a high school diploma or less were 1.2\% more likely to have a LBW (95\% CI: $1.00,1.02)$.

Significant predictor variables for PTB were a previous PTB, diabetes (insulin-controlled gestational onset and pre-existent diabetes), female infant, maternal anxiety during current pregnancy, maternal smoking and maternal narcotic and/or prescription drug use. Recent 
Table 2. Prevalence of clinical characteristics of women who had live singleton births at the LHSC between February 2009 and February 2014, $N=26,654$

\begin{tabular}{lrr}
\hline Maternal characteristic & $n$ & $\%$ \\
\hline Chronic hypertension & 507 & 1.9 \\
Diabetes & & 91.2 \\
None & 24,297 & 0.9 \\
Carbohydrate intolerance & 244 & 2.4 \\
Gestational onset, diet controlled & 637 & 1.8 \\
Gestational onset, insulin controlled & 479 & 0.9 \\
Previously diagnosed diabetes & 245 & \\
Risk behaviours & & 1.9 \\
Alcohol use & 511 & 17.0 \\
Drug use & 4521 & \\
Maternal drug use type & & 0.9 \\
Opioids & 253 & 0.2 \\
Narcotics & 66 & 0.3 \\
Cocaine & 9 & 0.0 \\
Hallucinogens & 9 & 2.2 \\
Marijuana & 576 & 0.1 \\
Amphetamines & 18 & 32.3 \\
Prescription drugs & 8617 & 2.8 \\
Natural health products & 752 & 0.9 \\
Methadone & 242 & 15.6 \\
Smoking & 4163 & 65.7 \\
Mother's primary language & & 0.1 \\
English & 17,522 & 34.2 \\
French & 23 & 667 \\
Other & & \\
\hline & &
\end{tabular}

immigration prevalence (OR: 0.99; 95\% CI: $0.96,0.99)$ and visible minority prevalence (OR: 0.99; 95\% CI: 0.99, 0.99) were significant. The odds ratio for previously diagnosed diabetes was the highest among all significant predictor variables, as women with this condition were 17.95 times more likely to have a PTB (95\% CI: $11.50,28.02)$.

Secondary analyses were performed utilizing birth percentiles less than the $5^{\text {th }}$ and $10^{\text {th }}$ percentiles and showed results that were similar to infants born LBW and PTB. The only SES variable significantly associated with birth $<5^{\text {th }}$ percentile was maternal education, with an odds ratio of 0.98 (95\% CI: 0.97, 0.99), although the relationship was weak and in the opposite direction as expected. Significant variables associated with birth weight $<10^{\text {th }}$ percentile included higher population density (OR: $1.06 ; 95 \% \mathrm{CI}$ : $1.03,1.1)$, chronic hypertension (OR: $2.22 ; 95 \% \mathrm{CI}: 1.48,3.34)$ and female infant (OR: 1.80; $95 \%$ CI: $1.54,2.11)$. Adjusted odd ratios for birth percentiles are shown in Table 4.

\section{Discussion}

The rates of LBW and PTB in the study sample were 6.4\% and 9.7\%, respectively. These rates are comparable to those reported by the Canadian Institute for Health Information 
Table 3. Adjusted odd ratios (aOR) and 95\% confidence intervals (CI) for final multivariable logistic regressions for predictors of LBW and PTB for women who had live singleton births at the LHSC between February 2009 and February 2014

\begin{tabular}{|c|c|c|}
\hline Characteristic & $\begin{array}{c}\text { LBW }^{\mathrm{a}} \\
\text { aOR }(95 \% \mathrm{CI})\end{array}$ & $\begin{array}{c}\text { PTB }^{\mathrm{b}} \\
\text { aOR }(95 \% \mathrm{CI})\end{array}$ \\
\hline \multicolumn{3}{|l|}{ Maternal characteristics } \\
\hline Number of previous PTBs & $1.32(1.06,1.66)$ & $2.10(1.84,2.41)$ \\
\hline Number of previous live births & $0.8(0.72,0.88)$ & - \\
\hline Chronic hypertension & $3.18(2.02,5.00)$ & $0.59(0.38,0.92)$ \\
\hline Diabetes (gestational onset, insulin controlled) & - & $2.42(1.64,3.57)$ \\
\hline Previously diagnosed diabetes & $0.34(0.16,0.70)$ & $17.95(11.50,28.02)$ \\
\hline Smoking & $2.43(1.88,3.15)$ & $0.59(0.49,0.70)$ \\
\hline Cardiac condition & - & $0.57(0.37,0.88)$ \\
\hline Marijuana use & $2.72(1.67,4.43)$ & - \\
\hline Narcotic use & - & $2.69(1.17,6.16)$ \\
\hline Prescription drug use & - & $1.36(1.19,1.57)$ \\
\hline Amphetamines use & $17.51(1.45,211.04)$ & - \\
\hline Anxiety this pregnancy & - & $1.41(1.07,1.86)$ \\
\hline BMI & $0.95(0.94,0.97)$ & - \\
\hline \multicolumn{3}{|l|}{ Infant characteristics } \\
\hline Gestational age at birth (weeks) & $0.34(0.32,0.36)$ & - \\
\hline Female infant & $1.64(1.34,2.02)$ & $0.55(0.48,0.63)$ \\
\hline Infant birth weight & - & $0.996(1.00,1.00)$ \\
\hline \multicolumn{3}{|l|}{ Neighbourhood characteristics } \\
\hline$\leq$ High school diploma & $1.01(1.00,1.02)$ & - \\
\hline Immigration prevalence & - & $0.99(0.96,0.99)$ \\
\hline Visible minority prevalence & - & $0.99(0.99,0.99)$ \\
\hline
\end{tabular}

${ }^{a}$ LBW model adjusted for all statistically significant univariate associations including: number of previous abortions, use of reproductive technology, distance to grocery store, immigration prevalence, after-tax LICO 2010, lone parent family prevalence, maternal age, maternal use of opioids, maternal use of methadone and maternal depression during this pregnancy.

${ }^{b}$ PTB model adjusted for all statistically significant univariate associations including: number of previous abortions, previous Caesarian section, reproductive technology use, distance to grocery store, maternal education, after-tax LICO 2010, maternal use of opioids, maternal use of cocaine, maternal use of marijuana, maternal use of amphetamines, maternal use of methadone and maternal depression during this pregnancy.

(CIHI) in 2010-2011, which found that 6.6\% of births in Canada were LBW and 8.1\% were PTB (Candian Institute for Health Information, 2011). The average maternal age in the present sample was $33.8 \pm 5.6$ years, slightly higher than the 2013 Ontario average age of mothers at the time of delivery of 30.5 years, and the Canadian average of 29.9 years (Statistics Canada, 2016). Smoking rates were also comparable to those found for Ontario. In this Southwestern Ontario sample, 15.6\% of women reported smoking during pregnancy, whereas $18.5 \%$ of pregnant women report smoking in Ontario (Cui et al., 2014). Similarly, the extent of socioeconomic disadvantage in the sample was also similar to what is typically found for the rest of Canada. Whereas $9 \%$ of Canadians were 
Table 4. Adjusted odd ratios (aOR) and 95\% confidence intervals (CI) for final multivariable logistic regression for predictors for birth weight percentiles $\left(<5^{\text {th }}\right.$ and $10^{\text {th }}$ percentiles) for women who had live singleton births at the LHSC between February 2009 and February 2014

\begin{tabular}{|c|c|c|}
\hline Characteristics & $\begin{array}{l}<5^{\text {th }} \text { percentile }^{\mathrm{a}} \\
\text { aOR }(95 \% \mathrm{CI})\end{array}$ & $\begin{array}{l}<10^{\text {th }} \text { percentile } \\
\text { aOR }(95 \% \text { CI })\end{array}$ \\
\hline \multicolumn{3}{|l|}{ Maternal characteristics } \\
\hline Number of previous PTBs & - & - \\
\hline Number of previous live births & $1.20(1.07,1.35)$ & - \\
\hline Number of previous term deliveries & - & $0.81(0.74,0.89)$ \\
\hline Chronic hypertension & - & $2.22(1.48,3.34)$ \\
\hline Diabetes (gestational onset, insulin controlled) & - & - \\
\hline Previously diagnosed diabetes & - & - \\
\hline Smoking & $0.47(0.37,0.61)$ & $1.65(1.36,2.01)$ \\
\hline Cardiac condition & - & - \\
\hline Marijuana use & $0.57(0.35,0.92)$ & $1.66(1.12,2.47)$ \\
\hline Narcotic use & - & - \\
\hline Prescription drug use & - & - \\
\hline Methadone use & $0.50(0.26,0.95)$ & - \\
\hline Amphetamine use & - & - \\
\hline Anxiety this pregnancy & - & - \\
\hline BMI & $1.04(1.02,1.05)$ & $0.97(0.95,0.98)$ \\
\hline \multicolumn{3}{|l|}{ Infant characteristics } \\
\hline Female infant & $0.57(0.46,0.70)$ & $1.80(1.54,2.11)$ \\
\hline Infant birth weight & - & - \\
\hline Gestational age at birth (weeks) & - & - \\
\hline \multicolumn{3}{|l|}{ Neighbourhood characteristics } \\
\hline$\leq$ High school diploma & $0.98(0.97,0.99)$ & - \\
\hline Immigration prevalence & - & - \\
\hline Visible minority prevalence & - & - \\
\hline Population density & - & $1.06(1.03,1.10)$ \\
\hline
\end{tabular}

${ }^{\mathrm{a}}$ Less than $5^{\text {th }}$ percentile model adjusted for all statistically significant univariate associations including: number of previous term deliveries, number of previous abortions, maternal chronic hypertension, maternal drug use, maternal cardiac condition, infant birth weight, maternal language, maternal mental health, after-tax LICO 2010, lone parent family prevalence, lone parent family-female-headed prevalence, population density, maternal use of opioids, maternal use of hallucinogens and maternal use of amphetamines.

${ }^{\mathrm{b}}$ Less than $10^{\text {th }}$ percentile model adjusted for all statistically significant univariate associations including: number of previous live births, previous Caesarian section, maternal drug use, infant birth weight, maternal mental health, immigration prevalence, visible minority prevalence, maternal education, after-tax LICO 2010, lone parent family prevalence, lone parent familyfemale-headed prevalence, population density and maternal use of cocaine.

below the after-tax LICO in 2010 (Statistics Canada, 2013), the median after-tax LICO in 2010 was $10.6 \%(4.5,22.3)$ in the present sample.

Results from the multivariate analyses suggest that the top risk factors for LBW were maternal amphetamine use, chronic hypertension and maternal marijuana use (OR 
respectively: $17.51,3.18,2.72$ ). Previously diagnosed diabetes, maternal narcotic use and insulin-controlled gestational diabetes were predictors of PTB (OR respectively: 17.95, $2.69,2.42$ ). Furthermore, it was also found that low levels of maternal education increased the likelihood of a LBW neonate, whereas immigration prevalence (OR: 0.99; $95 \%$ CI: $0.96,0.99)$ and visible minority prevalence (OR: $0.99 ; 95 \%$ CI: $0.99,0.99)$ were associated with lower odds of a PTB.

At this time, there has been minimal research conducted in Canada investigating SES and adverse birth outcomes. Although this study found a statistically significant association between maternal education and birth outcomes, the relationship was weak. Women were $1.2 \%$ more likely to have a LBW infant for every unit increase in having only a high school diploma or less, and maternal education was not associated with PTB. Conversely, in a nationally representative Canadian study, women without a high school diploma were 1.6 times more likely to have a PTB, but both maternal education and income had no impact on having a small-for-gestational-age infant (Lackman et al., 2001). Likewise, there was no impact of neighbourhood income on birth outcomes once adjusting for other covariates in the regression model. This suggests that the relationship between SES and birth outcomes may not be as strong as what is found in other developed countries, particularly the United States. It is possible that, relative to the United States, Canada's universal health care system provides a larger safety net for lower SES mothers and children.

While the association between maternal education and LBW was weak, this relationship is probably influenced by other maternal factors. Indeed, further bivariate analyses revealed that maternal education was significantly associated with smoking, BMI, drug use and anxiety (data not shown). This suggests a multifactorial influence on birth outcomes in Ontario, which has been supported by other investigations by this group (Campbell \& Seabrook, 2016). In addition, other unobserved variables that are also correlated with maternal education may lead to LBW. For example, low SES is likely to be associated with inadequate nutrition and delayed prenatal care, both of which could influence LBW.

This study is not without limitations. The study utilized an existing perinatal and neonatal database, and thus relied on maternal self-report and data collected from chart records. While the database included a large, population-based sample, it is possible that there may be incomplete, inconsistent or inaccurate data. For example, data on use of non-prescription drugs, alcohol, smoking and marijuana use may need to be interpreted with caution as they are probably under-reported. As well, median neighbourhood income was used as an indicator of socioeconomic attainment, and because there were no data on household income, it was not possible to correlate how strong neighbourhood income and household income were related. The association between mothers living below the after-tax low-income cut-off (i.e. mothers living in poverty) and birth outcomes was assessed, and this relationship attenuated to non-significance once other covariates were controlled in the model. Furthermore, there was no information available on how long mothers were residing in their neighbourhoods. The relationship between neighbourhood disadvantage and poor birth outcomes is much stronger the longer the mothers live in disadvantaged neighbourhoods (Collins et al., 2009). Nevertheless, the advantage of the present study is that many potential factors associated with birth outcomes, including socioeconomic status, demographics, prenatal factors and medical risks, were investigated using multivariate regression models. 
This study fills an important gap in the literature, as there have been limited Canadian studies investigating the association between SES and birth outcomes. There was a small, but statistically significant association between maternal education and low birth weight, whereas neighbourhood income had little impact on adverse birth outcomes. This suggests that, although risk factors for LBW and PTB are multifactorial, the aetiology of adverse birth outcomes does not appear to be strongly affected by SES. On the other hand, two risk factors that were strongly associated with LBW were maternal marijuana use and smoking tobacco during pregnancy. As these are modifiable risk factors, women who are pregnant or who are trying to get pregnant should be advised about the importance of marijuana and smoking cessation during the preconception period, or if presenting later, during pregnancy, to reduce the risk of having an adverse birth outcome.

\section{Acknowledgments}

This project was funded as a Graduate Studentship by the Department of Paediatrics, Schulich School of Medicine and Dentistry at the University of Western Ontario, London, Canada, and the authors thank them for their generous support. The authors also thank Dr Andrew Clark for his support and assistance with the Geographic Information System analysis.

\section{References}

Barker, D. J. P. (2004) The developmental origins of chronic adult disease. Journal of the American College of Nutrition 23(6), 588S-595S.

Behrman, R. E. \& Butler, A. S. (eds) (2007) Preterm Birth: Causes, Consequences, and Prevention. Institute of Medicine Committee on Understanding Premature Birth and Assuring Healthy Outcomes, National Academies Press, Washington, DC. URL: http://www.ncbi.nlm.nih.gov/ pubmed/20669423 (accessed 20th January 2017).

Boardman, J. D., Finch, B. K. \& Hummer, R. A. (2001) Race/ethnic differences in respiratory problems among a nationally-representative cohort of young children in the United States. Population Research and Policy Review 20(3), 187-206.

Campbell, E. E. \& Seabrook, J. A. (2016) The influence of socioeconomic status on adverse birth outcomes. Canadian Journal of Midwifery Practice and Research 15(2), 10-20.

Candian Institute for Health Information (2012) Highlights of 2009 - 2010 Selected Indicators Describing the Birthing Process in Canada. Health Indicator Reports. Canadian Institute for Health Information. URL: https://secure.cihi.ca/free_products/Childbirth_Highlights_201011_EN.pdf (accessed 20th January 2017).

Collins, J. W., Wambach, J., David, R. J. \& Rankin, K. M. (2009) Women's lifelong exposure to neighborhood poverty and low birth weight: a population-based study. Maternal and Child Health Journal 13(3), 326-333.

Cui, Y., Shooshtari, S., Forget, E. L., Clara, I. \& Cheung, K. F. (2014) Smoking during pregnancy: findings from the 2009-2010 Canadian community health survey. PLoS One 9(1), 1-5.

Daly, M. C., Duncan, G. J., Medonough, P. \& Williams, D. (2002) Optimal indicators of socioeconomic status for health research. American Journal of Public Health 92(7), 1151-1157.

Dunn, H. G. (1984) Social aspects of low birth weight. Canadian Medical Association Journal 130(9), 1131-1140.

Foulder-Hughes, L. A. \& Cooke, R. W. I. (2003) Motor, cognitive, and behavioural disorders in children born very preterm. Developmental Medicine and Child Neurology 45(2), 97-103. 
Haas, S. (2008) Trajectories of functional health: the "long arm" of childhood health and socioeconomic factors. Social Science \& Medicine 66(4), 849-861.

Healy, M. A. \& Gilliland, J. A. (2012) Quantifying the magnitude of environmental exposure misclassification when using imprecise address proxies in public health research. Spatial and Spatio-Temporal Epidemiology 3(1), 55-67.

Heaman, M., Kingston, D., Chalmers, B., Sauve, R., Lee, L. \& Young, D. (2013) Risk factors for preterm birth and small-for-gestational-age births among Canadian women. Paediatric and Perinatal Epidemiology 27(1), 54-61.

Hofman, P., Regan, F., Robinson, E., Knight, D., Jefferies, C., Jackson, W. \& Cutfield, W. (2004) Premature birth and later insulin resistance. New England Journal of Medicine 351, 2179-2186.

Joseph, K. S., Liston, R. M., Dodds, L., Dahlgren, L. \& Allen, A. C. (2007) Socioeconomic status and perinatal outcomes in a setting. Canadian Medical Association Journal 177(6), 3-10.

Kramer, M. S., Platt, R. W., Wen, S. W., Joseph, K. S., Allen, A., Abrahamowicz, M. \& Breart, G. (2001) A new and improved population-based Canadian reference for birth weight for gestational age. Pediatrics 108(2), e35.

Lackman, F., Capewell, V. \& Richardson, B. (2001) The risks of spontaneous preterm delivery and perinatal mortality in relation to size at birth according to fetal versus neonatal growth standards. American Journal of Obstetrics and Gynecology 184(5), 946-953.

Luo, Z. C., Kierans, W. J., Wilkins, R., Liston, R. M., Mohamed, J. \& Kramer, M. S. (2004) Disparities in birth outcomes by neighborhood income: temporal trends in rural and urban areas, British Columbia. Epidemiology 15(6), 679-686.

Luo, Z. C., Wilkins, R. \& Kramer, M. S. (2006) Effect of neighbourhood income and maternal education on birth outcomes: a population-based study. Canadian Medical Association Journal 174(10), 1415-1420.

Lynch, J. W., Kaplan, G. A. \& Salonen, J. T. (1997) Why do poor people behave poorly? Variation in adult health behaviours and psychosocial characteristics by stages of the socioeconomic lifecourse. Social Science \& Medicine 44(6), 809-819.

O'Rand, A. M. \& Hamil-Luker, J. (2005) Processes of cumulative adversity: childhood disadvantage and increased risk of heart attack across the life course. Journals of Gerontology, Series B 60B (Special Issue 11), 117-124.

Ross, N. A., Wolfson, M. C., Dunn, J. R., Berthelot, J. M., Kaplan, G. A. \& Lynch, J. W. (2000) Relation between income inequality and mortality in Canada and in the United States: cross sectional assessment using census data and vital statistics. British Medical Journal (Clinical Research Edition) 320(7239), 898-902.

Seabrook, J. A. \& Avison, W. R. (2010) Genotype-environment interaction and sociology: contributions and complexities. Social Science \& Medicine 70(9), 1277-1284.

Seabrook, J. A. \& Avison, W. R. (2012) Socioeconomic status and cumulative disadvantage for health outcomes. Canadian Review of Sociology 49(1), 50-68.

Seabrook, J. A. \& Avison, W. R. (2015) Family structure and children's socioeconomic attainment: a Canadian sample. Canadian Review of Sociology 52(1), 66-88.

Siqueira, F. M., Cota, L. O. M., Costa, J. E., Haddad, J. P. A., Lana, A. M. Q. \& Costa, F. O. (2007) Intrauterine growth restriction, low birth weight, and preterm birth: adverse pregnancy outcomes and their association with maternal periodontitis. Journal of Periodontology 78(12), 2266-2276.

Statistics Canada (2011) Census of Canada, 2011. Statistics Canada, Government of Canada.

Statistics Canada (2013) Persons in Low Income after Tax. Statistics Canada, Government of Canada. URL: http://www.statcan.gc.ca/tables-tableaux/sum-som/101/cst01/famil19a-eng.htm? sdi=low income (accessed $20^{\text {th }}$ January 2017).

Statistics Canada (2016) Trends in Canadian Births, 1993 to 2013. Statistics Canada, Government of Canada. URL: http://www.statcan.gc.ca/pub/82-625-x/2016001/article/14673-eng.htm (accessed 20th November 2016). 
Tucker, J. \& McGuire, W. (2004) Epidemiology of preterm birth. British Medical Journal (Clinical Research Edition) 329(7467), 675-678.

Williams, C. E., Davenport, E. S., Sterne, J. A., Sivapathasundaram, V., Fearne, J. M. \& Curtis, M. A. (2000) Mechanisms of risk in preterm low-birthweight infants. Periodontology 23, $142-150$.

Willson, A. E. (2009) 'Fundamental causes' of health disparities: a comparative analysis of Canada and the United States. International Sociology 24(1), 93-113.

World Health Organization (2014) Feto-Maternal Nutrition and Low Birth Weight. URL: http://www. who.int/nutrition/topics/feto_maternal/en/ (accessed 30th October 2014).

Zeitlin, J. A., Ancel, P. Y., Saurel-Cubizolles, M. J. \& Papiernik, E. (2001) Are risk factors the same for small for gestational age versus other preterm births? American Journal of Obstetrics and Gynecology 185(1), 208-215. 